Return to the Manage Active Submissions page at http://spie.org/app/submissions/tasks.aspx and approve or disapprove this submission. Your manuscript will not be published without this approval. Please contact author_help@spie.org with any questions or concerns.

\title{
Managing spatial diffraction through a periodic loss modulation
}

\author{
M. Botey, ${ }^{1,2}$ N.Kumar, ${ }^{2}$ R. Herrero, ${ }^{2}$ L. Maigyte, ${ }^{2}$ K.Staliunas ${ }^{2,3}$ \\ ${ }^{1}$ Departament de Física i Enginyeria Nuclear, Universitat Politècnica de Catalunya, EUETIB, \\ Compte Urgell 187, 08036 Barcelona, Spain \\ ${ }^{2}$ Departament de Física i Enginyeria Nuclear, Universitat Politècnica de Catalunya, Gaia, Rambla \\ Sant Nebridi 22, 08222 Terrassa, Spain \\ ${ }^{3}$ Institució Catalana de Recerca i Estudis Avançats (ICREA) \\ e-mail: muriel.botey@upc.edu
}

\begin{abstract}
We show that periodic distributions of gain or losses on the wavelength scale allow managing spatial diffraction of light beams, with no index contrast. It has been recently predicted that such artificial periodic structures, analogous to Photonic Crystals (PhCs), would also hold the novel spatial beam propagation effects reported for PhCs such as subdiffraction propagation, self-collimation, spatial filtering or beam focusing by a lens with flat interfaces. In particular, we consider an ideal periodic 2-dimensional (2D) arrangement of lossy cylinders embedded in air. We analytically show that this loss distribution affects diffraction. Indeed, a significant focusing behind a thin flat-flat crystal slab is observed, following the estimation of anomalous spatial dispersion for specific frequency ranges. Besides, close to the edges of the first Brillouin Zone, the light intensity map of a Gaussian beam exiting the lossy structure exhibits a high transmission windows instead of the transmission stop band expected for PhCs. This results from the strong anisotropic attenuation provided by the loss periodicity. Finally, we also consider a more realistic system with combined modulations of refractive index and losses: a 2D metallic photonic crystal (MPhC). We demonstrate that MPhCs also support selfcollimation and focusing, being such effects associated to zero and negative diffraction respectively. Finally, due to the anisotropic attenuation of light, the structure is also able to spatially filter noisy beams.
\end{abstract}

Keywords: photonic crystals, diffraction management, gain-loss modulation, metallic photonic crystal, scattering.

\section{INTRODUCTION}

Structured dielectric materials allow a precise control over both temporal and spatial dispersion of light; enabling the possibility to engineer the propagation of light beams, by managing diffraction. Among the reported novel effects are diffractionless propagation with no guiding boundaries or line defects $[1,2,3]$, negative diffraction which provides lenses with flat surfaces and hence insensible to alignment [4,5], or spatial filtering [6,7]. More recently, attention was paid to analogous artificial materials with a periodic Gain/Loss Modulation (GLM) on the wavelength scale, but with no refractive index contrast. It was predicted that such structures could hold sub-diffraction propagation, self-collimation or beam focusing [8,9]. As in the case of Photonic Crystals (PhC), such effects are accounted by the distortion of isofrequency contours of propagating modes in the wave-vector space. Additionally, in GLM materials, a strong anisotropic dependence of the wave amplification/attenuation arises, which could be used for applications such as beam spatial filtering $[10,11]$.

The present paper is devoted to analyze diffraction management associated with 2-dimensional (2D) loss modulated structures. We first consider the possibility to obtain focusing insensible to alignment from a purely Loss Modulated (LM) structure. The flat lensing effect arises from the compensation of negative phase-shifts, accumulated in propagation within the crystal, by normal diffraction beyond the structure. Such compensation determines the focal distance from the flat lens. We analytically determine the curvature of propagating modes in wave-vector space and then we numerically observe a significant focusing after a thin crystal slab. A deeper analysis on the phase profile curvature of the beam exiting the structure confirms a negative diffraction for given frequencies. Besides, contrary to what would be expected for a PhCs, the light intensity map of a Gaussian beam exiting the structure does not show a bandgap but a high 
Return to the Manage Active Submissions page at http://spie.org/app/submissions/tasks.aspx and approve or disapprove this submission. Your manuscript will not be published without this approval. Please contact author_help@spie.org with any questions or concerns.

transmission window, for frequencies close to the edges of the first Brillouin Zone; resulting from the anisotropic attenuation provided by the periodicity.[12]

Note, however, that both $\mathrm{PhC}$ and GLMs (or LM) are two ideal limiting cases since, in general, the refractive index and the gain/loss variations are intrinsically related via the Kramers-Kronig relations. Therefore, we perform a detailed analysis of propagation $\mathrm{f}$ light beams in a similar arrangement of gold cylinders embedded in air. Negative diffraction has been already predicted in metallic PhCs (MPhCs) $[13,14]$. We here show that MPhC support nondiffractive propagation associated to zero diffraction. Moreover, negative diffraction enables focusing of light beams after propagating through a finite $\mathrm{MPhC}$ slab. Finally, we demonstrate that MPhCs are also able to spatially filter noisy beams, due to the anisotropic attenuation of light. [15]

\section{IDEAL LOSS MODULATION}

\subsection{Model. Coupled-mode expansion.}

In order to determine the curvature of the spatial diffraction, we use a coupled-mode expansion. We start from the wave equation for a monochromatic wave with a sinusoidal modulation of the refractive index:

$$
\nabla^{2} E+n^{2} \frac{\omega}{c^{2}} E=0, \quad n=n_{0}+2 n_{1}\left[\cos \left(\vec{q}_{1,0} \vec{r}\right)+\cos \left(\vec{q}_{0,1} \vec{r}\right)\right]
$$

where $n_{0}$ is the homogenous refractive index and $n_{1}$ the amplitude of the modulation. The geometry is fixed by the reciprocal lattice vectors $\vec{q}_{1,0}=q(\sin \beta, \cos \beta)$ and $\vec{q}_{0,1}=q(-\sin \beta, \cos \beta)$. When expand the electric field in terms of the first harmonics of the modulation, $\vec{k}_{l, p}=\vec{k}+l \vec{q}_{0,1}+p \vec{q}_{1,0}$ :

$$
E=\sum_{l, p}\left[A_{l, p} e^{i \vec{k}_{l, p} \vec{r}}\right]
$$

We substitute eqs.1,2 into eq.3, to obtain a coupled system of equations of all modes:

$$
\sum_{l, p}\left[\nabla^{2} A_{l, p}+2 i \vec{k}_{l, p} \vec{\nabla} A_{l, p}-k_{l, p}^{2}+n^{2} k_{0}^{2} A_{l, p}\right] e^{i \vec{k}_{l, p} \vec{r}}=0
$$

where $A_{l, p}$ stands for the amplitudes of each mode in the field expansion, and $k_{0}=2 \pi / \lambda$. Without loss of generality, consider a rhombic structure, being the angle between the two lattice vectors $2 \beta=105^{\circ} \quad$ - note that $2 \beta=90^{\circ}$ for a square geometry and $2 \beta=120^{\circ}$, for a triangular lattice-. For small frequencies it is sufficient to consider the four modes $(l, p)=(0,0),(1,0),(0,1)$ which overlap at the edge of the First Brilloiun Zone (FBZ). We solve the coupled equations for different incident wavelengths and obtain the corresponding complex eigenvalues or wave-vectors, $k$ for each mode. We assume $n_{1}=0.09 \mathrm{i}$ and $n_{0}=1+0.2 \mathrm{i}$; hence, a LM structure.

a)

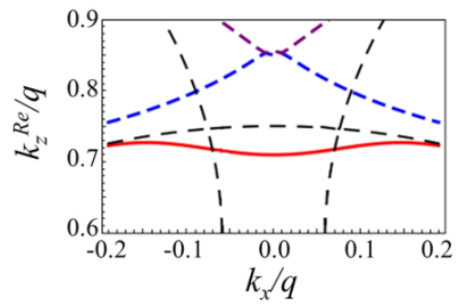

b)

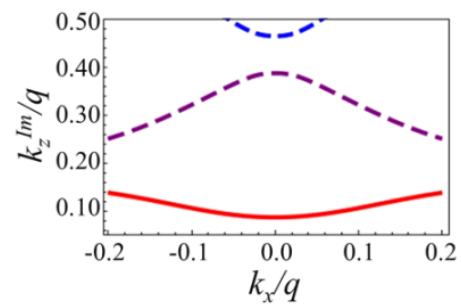

c)

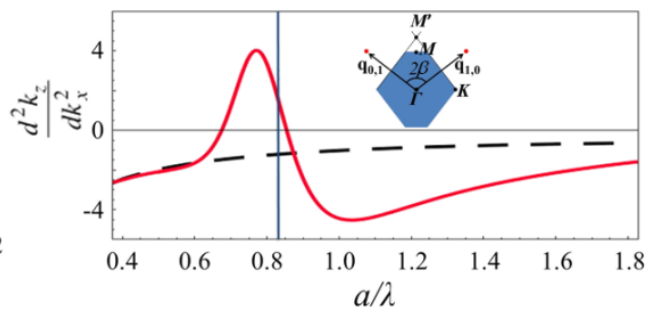

Figure.1. Real and imaginary a)/b) dispersion of the first three modes for a rhombic harmonic LM structure with $2 \beta=75^{\circ}$, for $a / \lambda=0.76$. c) Curvature of the dispersion for the least attenuated mode at $k_{x}=0$. The $M^{\prime}$ point lays at $a / \lambda=0.83$. 
Return to the Manage Active Submissions page at http://spie.org/app/submissions/tasks.aspx and approve or disapprove this submission. Your manuscript will not be published without this approval. Please contact author_help@spie.org with any questions or concerns.

The real part of the wave-vector describes the spatial dispersion which for given frequencies exhibits an anomalous curvature, see Fig. 1a. On the other hand, the imaginary part determines attenuation. Figure 1c depicts the curvature of diffraction through the second derivative of the in-axis spatial longitudinal dispersion, $\frac{d^{2} k_{z}}{d k_{x}^{2}}$ at $k_{\mathrm{x}}=0$. If we only plot the dominant mode, which corresponds to the less attenuated mode -with a smaller imaginary part of the wave-vector-, we see that it strongly depends on frequency, especially close to the edge of the FBZ. As expected, for small frequencies the curvature of the dominant mode is concave, while we observe a frequency range where this curvature becomes convex, and hence leads to anomalous diffraction. The beam suffers from normal diffraction and therefore curvature is concave, negativejust before the M' point, see Fig.1c. Analogous results can be found considering other geometries [12].

\subsection{Numerical Analysis}

In simulations, we consider a finite-sized 2D periodic structure made of absorbing cylinders embedded in air. The refractive index contrast is considered to be zero and cylinders complex refractive index is taken $1+0.4 \mathrm{i}$ (absorption coefficient of $5 \cdot 10^{4} \mathrm{~cm}^{-1}$ for $\lambda=1 \mu \mathrm{m}$ ), see Fig. 2a. We propagate a monochromatic linearly polarized - electric field parallel to the rods- Gaussian beam along the $\Gamma M$ direction of the rhombic LM structure using Finite Difference Time Domain (FDTD), and we obtain the intensity transmission map as depicted in Fig.2.a. The transmitted intensity, plotted in Fig. $2 \mathrm{~b}$ as a function of the beam carrier frequency, exhibits a high transmission window around the edge of the FBZ, $a / \lambda \approx 8$. Note that such high intensity transmission band, determined for a LM structure lays, precisely, where a low intensity band or bandgap would be expected in the case of a photonic crystals, i.e. in the case that the real part of the refractive index would be modulated -instead of the imaginary one-. However, more important, inspecting a cross section of such intensity map, shown in Fig.2c, at a given distance from the sample, we observe the expected focalization predicted by negative diffraction inside the structure. We may compare the exiting beam with a similar beam propagated through air and we clearly see the effect, plotted in Fig. 2c as a reference.
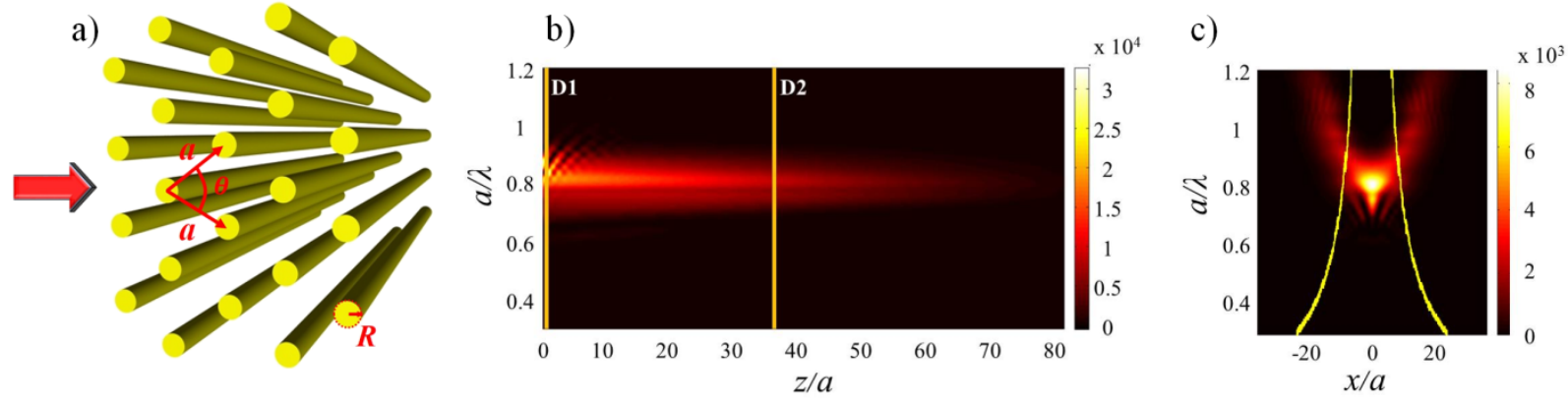

Figure. 2. a) Schematic representation of a lattice made of absorbing cylinders embedded in air with $\theta=75^{\circ}-$ which corresponds to $2 \beta=105^{\circ}$ in reciprocal space- and $R=0.2$, where $a$ is the direct lattice distance, $a=2 \pi / q$. b) FDTD transmission map for cylinders with $n_{c y l}=1+0.4 i$, the vertical axis denotes the carrier frequency, in $a / \lambda$ units, of the $1.5 \mu \mathrm{m}$ wide incident Gaussian and the horizontal axis the normalized distance from the sample, D1 is the end of the structure. b) Transverse cross section at a distance of 36 a after the structure, denoted by D2 in figure a), where the continuous the yellow curve is for comparison with propagation in free space.

A deeper analysis can be performed on the obtained numerical results. We calculate the second derivative of the phase of the spatial Fourier transform of transmitted beams, just after the structure, for different frequencies. The evolution of such phase curvature is reproduced in Fig. 3a, as a function of frequency. For frequencies far below the $M$ point the phase profile on a vertical plane after the sample has a concave curvature, hence indicating normal diffraction, see Fig.3a. However, as shown in Fig. $3 b$, approaching $a / \lambda \approx 83$ the phase curvature becomes positive, corresponding to a convex wavefront. In this case, the phase accumulated by negative diffraction in the structure is compensated after propagating through free space and focalized after the LM structure. In addition, for frequencies nearing the resonance condition, the spatial components around $k_{\mathrm{x}}=0$ have a minimum attenuation. Hence, less attenuation in combination with negative refraction inside the LM material yields to a high intensity transmission window. For higher frequencies, after the FBZ edge, diffraction is again normal and the phase curvature recovers its concave shape, see Fig. 3c. 
Return to the Manage Active Submissions page at http://spie.org/app/submissions/tasks.aspx and approve or disapprove this submission. Your manuscript will not be published without this approval. Please contact author_help@spie.org with any questions or concerns.

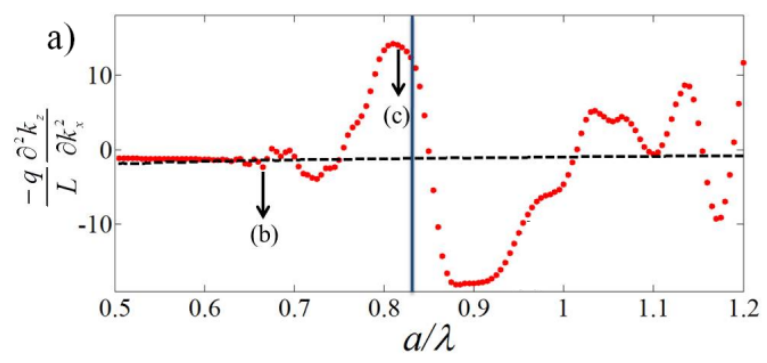

b)

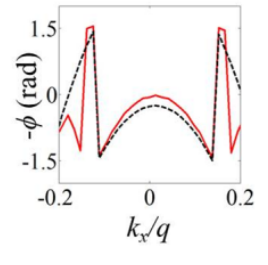

c)

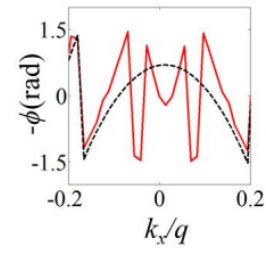

Figure. 3. a) Curvature of the phase of the spatial Fourier transform of a Gaussian beam just exiting a LMM slab of length L (solid curve), compared with the normal diffraction concave curvature (dashed curve) of the same beam propagated in free space, for $\mathrm{a} / \lambda=0.67$ and 0.81 respectively. $\mathrm{b}$ )/c) Comparison of the phase curvatures of both beams for two specific frequencies.

\section{METALLIC PHOTONIC CRYSTALS}

In this section we consider a realistic system a $2 \mathrm{D} \mathrm{MPhC} \mathrm{made} \mathrm{of} \mathrm{gold} \mathrm{cylinders} \mathrm{in} \mathrm{air} \mathrm{analogous} \mathrm{to} \mathrm{the} \mathrm{structure}$ previously considered, shown in Fig. 1a with $a=0.83 \mu \mathrm{m}$. For frequencies larger than the plasma frequency, $\omega_{p}$, the dielectric constant of gold is estimated by the Drude model, therefore following the expression: $\varepsilon(\omega)=1-\omega_{p}^{2} /(\omega(\omega-i \gamma))$, where $\omega$ is the frequency of light, and $\gamma$ stands for the collision frequency of gold $\left(\omega_{p}=12229.7 \mathrm{THz}, \gamma=139.7 \mathrm{THz}\right)$. We analyze propagation of a monochromatic Gaussian beam along the $\Gamma \mathrm{M}$ direction, normally incident on the structure. The expected propagation effects may be explained by the curvature of the spatial dispersion curves or the isofrequency contours in wave-vector space.

\subsection{Nondiffractive propagation}

In the calculated spatial dispersion for the structure above described, a flattening of the dispersion isofrequency contour can be observed for $a / \lambda=0.68(f=246 \mathrm{THz})$, indicating a non-diffractive propagation inside the MPhC, see Fig. $4 \mathrm{a}$. Therefore, a Gaussian beam with central frequency $246 \mathrm{THz}$ supports nondiffractive propagation inside the structure, as shown in Fig. 4b. The intensity in Fig. 4a is normalized at each cross-section (vertical cross section, $z=$ const.) for a better visualization of the effect since, the beam intensity exponentially decreases in propagation along the structure due to losses and reflections. Figure 3.b depicts such on-axis beam intensity (horizontal cross section at $x=0$ ) in logarithmic scale. Note, in Fig. 4a, that for higher frequencies, the dispersion curve becomes convex, which would cause the beam to undergo negative diffraction.
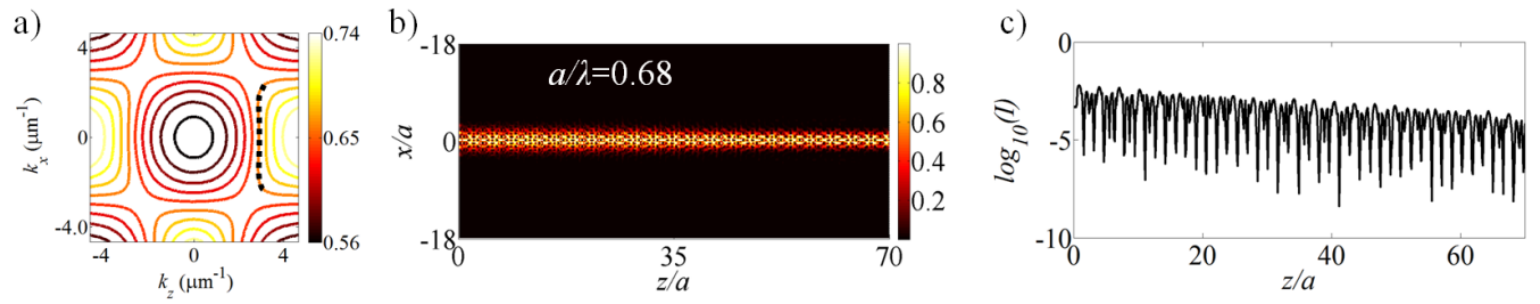

Figure. 4. Non-diffractive propagation inside the infinite $\mathrm{MPhC}$ with the same structural parameters described above. b) Normalized intensity profile of the propagated beam in a length of $70 a$ considering an initial beam of width $2.5 a$. at $a . / \lambda=0$. c) Horizontal cross section intensity profile corresponding to $x=0$. 


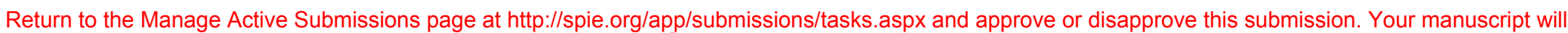
not be published without this approval. Please contact author_help@spie.org with any questions or concerns.

\subsection{Focalization by a flat MPhC}

Figures 5a,b,c shows the focusing properties of a MPhC slab for different frequency, when compared to free space propagation. Precisely at those frequencies, slightly above resonance, the isofrequency contours display anomalous curvature, see Fig. 5d. We clearly observe focalization of the transmitted beams after free propagation in air. The phase accumulated by negative diffraction within the structure is compensated by positive diffraction in air. The focal point corresponds to the position where and all angular components of the beam are in phase. Hence, the position of the focal point, as well as intensity at this focal point, is determined by the curvature of the spatial dispersion, and it is strongly frequency dependent.

a)

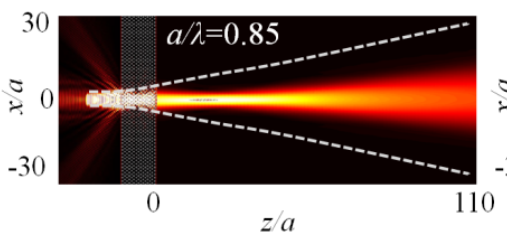

b)

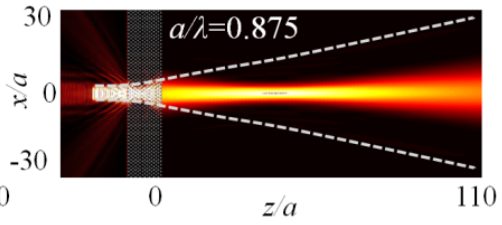

c)

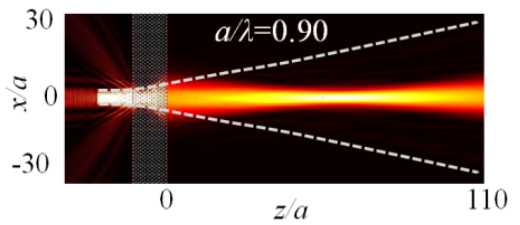

d)

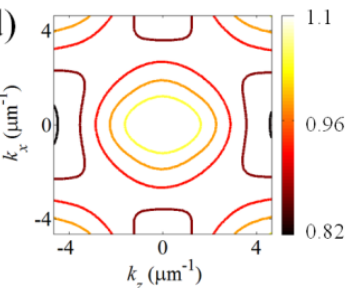

e)

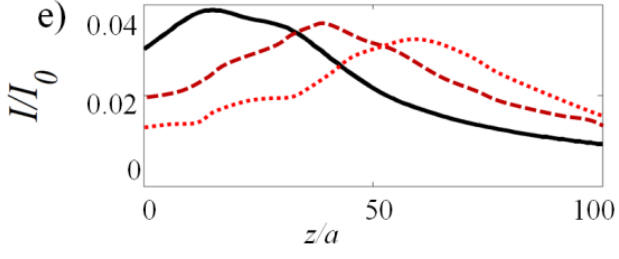

Figure. 5. Focusing behind $\mathrm{MPhC}$ with the same parameters as in Fig.4. a),b),c) Display the field intensity profile corresponding on-axis intensity (horizontal cross section) in free space propagation behind the structure at the position $x=0$, for a monochromatic Gaussian beam at different frequencies; $a . / \lambda=0.85,0.875$, and $0.9(f=307,316$, and 325 $\mathrm{THz}$ ). The dashed line is for comparison with free space propagation. d) Isofrequency contour for frequencies above resonance. e) Maximum intensity (at the focal point) behind the MPhC depending on frequency.

The focal position in Figs. 5a,b,c can be clearly identified with the maximum of the on-axis horizontal cross section of the corresponding beam intensity behind the structure, as shown in Figs. 5e where intensity is normalized to the intensity of the initial beam. The more curved the dispersion segment, the more distant the focal point. A larger negative diffraction accumulated within the structure, requires a longer propagation distance to compensate it by positive diffraction in a homogeneous material. FDTD calculations clearly confirm that focalization is observed at frequencies for which negative diffraction occurs in the structure, which are frequencies close resonance. As expected in this case, higher frequencies correspond to larger focal distances as the curvature of dispersion increases. The maximum intensity of the transmitted beam, calculated either at the focal point or just on the right facet of the MPhC slab, as a function of frequency, is summarized in Fig. 6a-normalized to the intensity of the incident beam.

a)

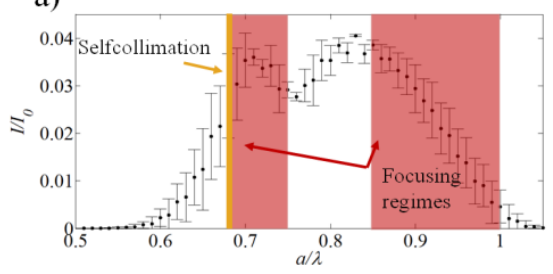

b)

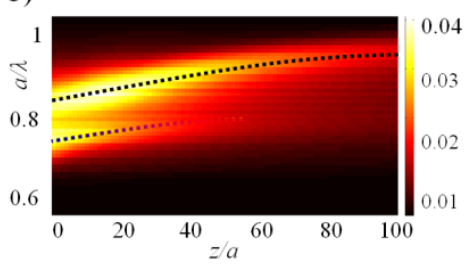

c)

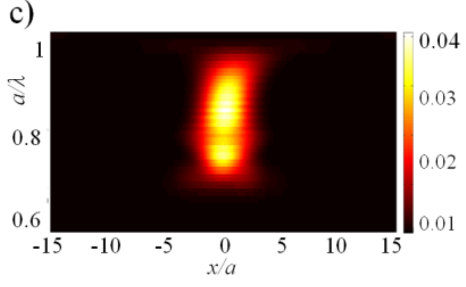

Figure. 6. a) Maximum intensity beyond the MPhC (either at the output facet of the MPhC slab or at the focal point) depending on frequency. b) Normalized intensity distribution along the horizontal axis, $z$, depending on frequency . The focal distance from the MPhC is indicated by dark dashed lines. c) Normalized intensity map at the focal plane depending on frequency. 
Return to the Manage Active Submissions page at http://spie.org/app/submissions/tasks.aspx and approve or disapprove this submission. Your manuscript will not be published without this approval. Please contact author_help@spie.org with any questions or concerns.

The highest intensities obtained around $a / \lambda=0.7$ and at $a / \lambda=0.85$ can be attributed to two different situations. On one hand, $a / \lambda=0.68$ the nondiffractive, propagation along the crystal (Fig. 4) provides a high transmission just at the crystal output. For $a / \lambda>0.68$, we find a focusing regime and the beam maximum intensity separated from the output facet, increasing with frequency. For $a / \lambda=0.85$ a new focusing regime is found and intensity at focus reduces as frequency increases, due to the width of isofrequency contours which limits focusing to a few angular beam components. Inspecting in more detail Fig. $6 \mathrm{~b}$ we again appreciate the two key frequency ranges. One, around $a / \lambda=0.68$ which corresponds to a collimated propagation and a first focusing range, and another from $a / \lambda=0.85$ (just above resonance) Fig. $6 \mathrm{c}$, representing the intensity profile on a transversal plane at the minimum beam width position, beyond the crystal, exhibits again the focusing frequency ranges $(a / \lambda=0.68-0.74$ and $a / \lambda=0.85-1.0)$.

\subsection{Spatial filtering of a noisy beam}

While the curvature of the isofrequency curves defines the focal distance, the size of the isofrequency contour itself is also important. The smaller it is, the smaller the angular range of beams components is affected. Hence, less beam components can be focused or, eventually, propagated through the structure. In other words, the size of the isofrequency curves determines the spatial (angular) filtering of the structure. The focusing performance for narrower beams is weaker than for broader ones. This can be seen as a signature of spatial filtering in a MPhC. Therefore, either narrow or noisy beams exhibiting an angular spectrum broader than the corresponding contour, will be spatially (angularly) filtered while propagating trough the structure. The narrower the beam, the wider the angular range of filtered out spatial components.

Figure 7 shows the transmission of noisy beams by a MPhC. We introduce noise by propagating Gaussian beams through random diffusers. Figures $7 \mathrm{a}, \mathrm{b}$ display the comparison of the transmission of such a randomized beam and its free space propagation. It is clear that, after propagation trough the structure, the spatial quality of the beam increases significantly. The filtered beam displays a smooth shape after propagation through the $\mathrm{MPhC}$, as shown in Fig. 7. c. Finally, the intensity width profile of the filtered beam is comparable to the input noisy beam, while the reference free propagated beam becomes broader than the incident one.

a)

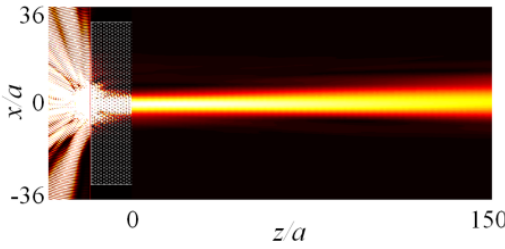

b)

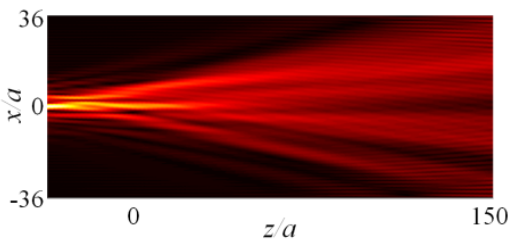

c)

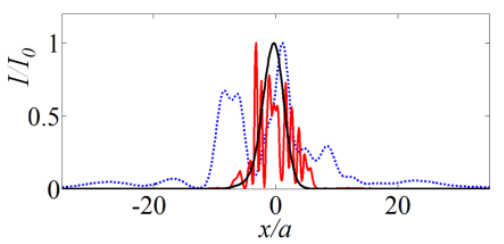

Figure. 7. a) Propagation of a random input beam in a MPhC and behind it with frequency a/ $\lambda=0.76$. b) Propagation of the same random input beam in free space without the MPhC structure. c) Normalized intensity profiles of the transmitted filtered beam (solid black), compared to the incident-randomized beam (red) and to the same beam propagated in free space (dotted).

\section{CONCLUSIONS}

While the drawback of most periodic structures are losses, as part of light is absorbed, we point out that loss modulations provide new perspectives in light manipulation on the micrometer scale. We investigate beam shaping effects by a rhombic 2D periodic loss distribution. We show that such structure holds negative diffraction accounted by convex negative diffraction spatial dispersion segments propagation. Therefore, negative diffraction can be compensated in free propagation, leading to focalization. In particular, we analyze MPhCs made of gold cylinders embedded in air. Nondiffractive propagation through the structure is demonstrated; the spatial shape of the beams is kept constant for frequencies with flat spatial dispersion segments. For given frequency ranges, we also observe a clear focalization of the beams beyond a thin flat MPhC slab; and we prove that the focal position is clearly explained by the curvature of the spatial dispersion segments. Finally, we investigate on the spatial filtering performance of this same structure. The predicted phenomena are expected to be generic for spatially modulated materials and other kinds of waves. Indeed, we extend our work to acoustics and obtain similar results from phononic crystals with periodically distributed losses.[16] 
Return to the Manage Active Submissions page at http://spie.org/app/submissions/tasks.aspx and approve or disapprove this submission. Your manuscript will not be published without this approval. Please contact author_help@spie.org with any questions or concerns.

\section{ACKNOWLEDGEMENTS}

The work was financially supported by Spanish Ministerio de Educación y Ciencia and European Union FEDER through project FIS2011-29731-C02-01.

\section{REFERENCES}

[1] H. Kosaka, and T. Kawashima, A. Tomita, M. Notomi, T. Tamamura, T. Sato, S. Kawakami, "Self-collimating phenomena in photonic crystals," Appl. Phys. Lett. 74, 1212-1214 (1999).

[2] R. Illiew, C. Etrich, U. Peschel, F. Lederer, M. Augustin, H. J. Fuchs, D. Schelle, E. B. Kley, S. Nolte, and A. Tunnermann, "Diffractionless propagation of light in a low-index photonic-crystal film," Appl. Phys. Lett. 85, 58545856 (2004).

[3] K. Staliunas, and R. Herrero, "Nondiffractive propagation of light in photonic crystals," Phys. Rev. E 73, 016601 (2006).

[4] J. B. Pendry, "Negative Refraction Makes a Perfect Lens," Phys. Rev. Letters 85, 3966 (2000).

[5] P. V. Parimi, W.T. Lu, P. Vodo, and Srinivas Sridhar, "Photonic crystals: Imaging by flat lens using negative refraction," Natutre 426, 404 (2003).

[6] K. Staliunas, R. Herrero, and R. Vilaseca, "Subdiffraction and spatial filtering due to periodic spatial modulation of the gain-loss profile," Phys. Rev. A. 80, 013821 (2009).

[7] L. Maigyte, T. Gertus, M. Peckus, J. Trull, C. Cojocaru, V. Sirutkaitis, and K. Staliunas, "Signatures of light-beam spatial filtering in a three-dimensional photonic crystal,” Phys. Rev. A. 82, 043819 (2010).

[8] M. Botey, R. Herrero, and K. Staliunas, "Light in materials with periodic gain/loss modulation on a wavelength scale," Phys. Rev. A. 82, 013828 (2010).

[9] N. Kumar, M. Botey, R. Herrero, Y. Loiko, and K. Staliunas, "High-directional wave propagation in periodic loss modulated materials," Phot. Nano. Fund. Appl. 10(4), 644-650 (2012).

[10] M. Radziunas, M. Botey, R. Herrero, and K. Staliunas,"Intrinsic beam shaping mechanism in spatially modulated broad area semiconductor amplifiers," Appl. Phys. Lett. 103, 132101 (2013).

[11] N. Kumar, L. Maigyte, M. Botey, R. Herrero, and K. Staliunas, "Flat Beam shaping in two-dimensional metallic photonic crystals," JOSA B, 31, 686-690 (2014).

[13] M. A. Kaliteevski, S. Brand, J. Garvie-Cook, R. A. Abram, and J. M. Chamberlain, "Terahertz filter based on refractive properties of metallic photonic crystal," Opt. Exp., 16, 7330-7335 (2011).

[14] G. P. Swift, A. J. Gallant, N. Kaliteevskaya, M. A. Kaliteevski, S. Brand, D. Dai, A. J. Baragwanath, I. Iorsh, R. A. Abram, and J. M. Chamberlain, "Negative refraction and the spectral filtering of terahertz radiation by a photonic crystal prism," Opt. Let. 36, 1641-1643 (2011).

[15] N. Kumar, R. Herrero, M. Botey, and K. Staliunas, "Flat lensing by periodic loss-modulated materials," JOSA B. 30, 2684-2688 (2014).

[16] A. Cebrecos, R. Pico, V. Romero-Garcia, A.M. Yasser, L. Maygite, R. Herrero, M. Botey, V. J. Sanchez-Morcillo, and K. Staliunas "Antibandgaps in loss modulates media". In preparation. 